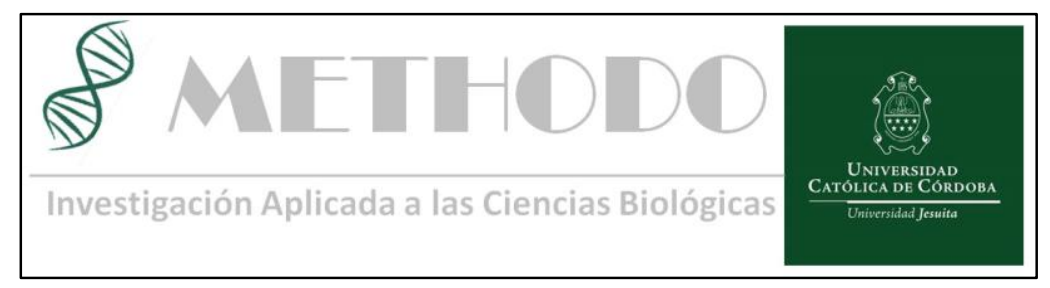

\title{
Tos crónica por hipersensibilidad refleja: un nuevo paradigma con implicancias diagnósticas y terapéuticas
}

\section{Cough hypersensitivity syndrome: a new paradigm with diagnostic and therapeutic implications.}

\author{
Ricardo J. Saranz ${ }^{1}$, Alejandro Lozano ${ }^{1}$, Natalia A Lozano ${ }^{1}$, Graciela Alegre ${ }^{1}$. \\ 1 Universidad católica de Córdoba, Argentina, Facultad de Ciencias de la Salud, Cátedra de Inmunología. Clínica Universitaria Reina Fabiola, Servicio de Alergia \\ e Inmunología. \\ Los autores declaran que este trabajo de revisión se realizó con financiación de la Secretaría de Investigación y Vinculación Tecnológica de la Universidad Católica \\ de Córdoba. Correspondencia: Ricardo J. Saranz. Servicio de Alergia e Inmunología- Clínica Universitaria Reina Fabiola. Oncativo 1248 -X5004F \\ HP- Córdoba, Argentina; e-mail: rsaranz@arnet.com.ar.
}

\section{Resumen}

La tos es un reflejo protector del aparato respiratorio que si se prolonga en el tiempo puede afectar seriamente la calidad de vida. Recientemente un nuevo paradigma califica a la tos por hipersensibilidad refleja consecuente a una exagerada sensibilidad de las terminaciones nerviosas periféricas a diversos estímulos y a alteraciones en los núcleos centrales integradores del reflejo. Este nuevo paradigma tiene implicancias terapéuticas, más allá del tratamiento de la causa de la tos. En este artículo se revisarán los nuevos mecanismos implicados en la tos por hipersensibilidad refleja y las nuevas drogas en investigación que modulan el reflejo tusígeno. A pesar de las nuevas investigaciones farmacológicas se necesitan evidencias más seguras para aconsejar un tratamiento "sintomático" de estos pacientes que contribuya al control de la hipersensibilidad central y periférica del reflejo de la tos.

\section{Abstract}

Cough is a protective reflex of the respiratory system that if persist over time can seriously affect the quality of life. Recently, a new paradigm qualifies cough as a consequence of a reflex hypersensitivity resulting from an exaggerated sensitivity of the peripheral nerve endings to various stimuli and central neural alterations in cough processing. This new paradigm has therapeutic implications, beyond the causal treatment of cough. In this article we will review the new mechanisms involved in cough due to reflex hypersensitivity and new therapeutic strategies under development that modulate the cough reflex. Despite the new pharmacological investigations, safer evidence is needed to advise a "symptomatic" treatment of these patients that contributes to the control of central and peripheral hypersensitivity of the cough reflex.

\section{Introducción}

La tos implica una modificación del patrón respiratorio normal y representa un reflejo protector del aparato respiratorio ante agentes químicos, mecánicos y térmicos. Por sus características y duración, puede transformarse en un síntoma molesto que afecta la calidad de vida de las personas ${ }^{1,2}$. Es esencialmente un síntoma y no una enfermedad, por lo que el objetivo médico prioritario es identificar las causas respiratorias y extra-respiratorias que la ocasionan, para su tratamiento etiológico adecuado. 
Es necesario clasificar a la tos por su duración con fines clínicos, diagnósticos y terapéuticos en: tos aguda ( $<3$ semanas), tos subaguda (3-8 semanas), y tos crónica ( $>8$ semanas) ${ }^{3}$. Generalmente las dos primeras formas reconocen un origen infeccioso viral o bacteriano y en la mayoría de los casos tiene resolución espontánea o tratamiento específico ocasional ${ }^{4}$. En otros casos puede ser la primera manifestación de una futura enfermedad que tiene en la tos una expresión más prolongada ${ }^{5}$. Entre un 5 y $30 \%$ de la población padece de tos crónica ${ }^{6,7}$ lo que implica un fuerte impacto sanitario y un desafío diagnóstico para médicos de distintas especialidades 8,9 . El objetivo de esta revisión es recopilar las más recientes evidencias en la fisiopatología del reflejo tusígeno que podrían justificar un aumento de la sensibilidad refleja responsable de la prolongación de la tos y sus nuevas posibilidades terapéuticas más allá del tratamiento etiológico de este complejo síntoma.

\section{Material y métodos}

Se realizó una búsqueda bibliográfica, en idioma inglés, en base de datos electrónica Medline, considerando como términos de búsqueda cough, hypersensitivity, cough réflex, chronic refractory cough, unexplained cough, pathophysiology, cough suppressants, antitussive y cough treatment de los últimos cinco años (2013-2018). Bibliografía previa a dicho intervalo de tiempo sirvió de utilidad complementaria. Los autores realizaron una revisión crítica de cada uno de los artículos que figuran en referencias.

\section{El reflejo de la tos}

El mecanismo reflejo de la tos es muy complejo y está estructurado como un circuito neurológico que conecta receptores periféricos con el sistema nervioso central ${ }^{10}$. Consiste de cinco componentes: a) receptores de la tos; b) nervios aferentes; c) centro nervioso integrador, d) nervios eferentes y e) músculos efectores ${ }^{10,11}$ (figura 1). Los receptores se distribuyen a lo largo de todo el tracto respiratorio y en otras localizaciones como oído externo, estómago, esófago, pericardio y diafragma. Existen receptores irritativos de rápida adaptación (principalmente concentrados en la vía aérea de mayor calibre en el lado luminal de la membrana basal), receptores mecánicos sensibles a cambios en el calibre de la vía aérea y receptores químicos sensibles a gases y humos.

Las vías sensoriales aferentes discurren principalmente por los nervios vago y trigémino en dos suptipos de fibras ${ }^{12}$. El subtipo de fibras-c sensoriales forman una red de fibras no mielinizadas (de lenta conducción), son sensibles a la capsaicina, irritantes químicos, térmicos, ácidos y proinflamatorios, por activación de canales iónicos del potencial transitorio del receptor vanilloide tipo 1(TRPV1) y potencial transitorio del receptor ankirina tipo 1 (TRPA1) ${ }^{11}$. El segundo subtipo, se corresponde con fibras $\mathrm{A} \delta$, mielinizadas de ubicación subepitelial en vías aéreas proximales, de rápida conducción y que responden a estímulos mecánicos, ácidos y osmolares, pero que no expresan TRPV1 y no son sensibles a la estimulación con capsaicina 12,13. Otros receptores vágales, menos importantes incluyen los mecanoreceptores intrapulmonares de adaptación lenta y rápida que despliegan una actividad rítmica para la regulación de la respiración eupneica. Estas fibras no disparan tos en forma directa, pero pueden modular la desencadenada desde las principales vías aferentes mencionadas más arriba ${ }^{12}$. Los componentes nerviosos centrales del reflejo tusivo han sido investigados en modelos animales y humanos 14,15. Los estímulos de estas vías aferentes se integran en el núcleo solitario y el paratrigeminal en el tronco cerebral activando, eventualmente, una compleja red neural que se transmite a las zonas cortical y subcortical responsables de la sensación de irritación de la vía aérea y necesidad urgente de toser. De allí surge la respuesta eferente-efectora que es transmitida hacia los músculos espiratorios y diafragma por el nervio motor espinal y el frénico y hacia la laringe por las ramas recurrentes del vago. Terminaciones del parasimpático abastecen a la tráquea y los bronquios y por su efecto de contracción del músculo liso contribuyen al esfuerzo de la tos por estrechamiento de la vía aérea y consecuente aumento de la velocidad del flujo ${ }^{10}$

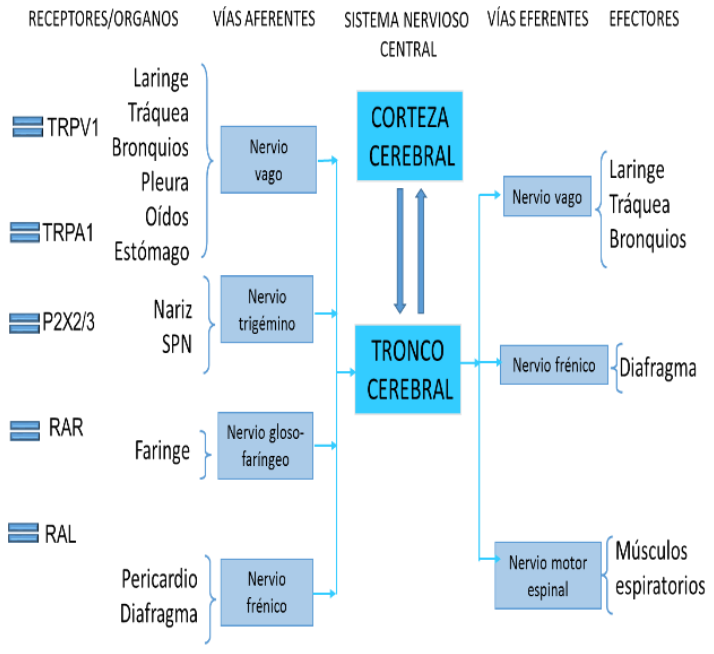




\section{El paradigma del diagnóstico etiológico y sus debilidades}

Desde la década del noventa se introdujo un protocolo diagnóstico anatómico teniendo en cuenta la distribución de los receptores de la tos y las características anatómicas del reflejo tusígeno ${ }^{16}$. Esto significó un gran avance en el tratamiento de la tos basada en la etiología bajo el paradigma "identificar la causa y tratarla" desaconsejando el solo abordaje del síntoma ${ }^{3,16,17 .}$ Así, con algunas diferencias relacionadas a la edad, la mayoría de los protocolos emanados de revisiones y consensos consideran que las tres principales causas de tos crónica son rino-sinusitis (tos asociada a patología de vía aérea superior), vía aérea inferior hiperreactiva (tos "asma equivalente") y enfermedad asociada a reflujo gastroesofágico 3,4,17-21. Sin embargo, estudios posteriores comprobaron que no todos los pacientes que padecen esas enfermedades manifiestan tos crónica como síntoma prevalente 22,23 . Por otro lado, el tratamiento específico de la causa no siempre es exitosa para el control de la tos: un 15 a $30 \%$ de esos pacientes persisten con tos, a pesar del tratamiento etiológico, incorporándose a la categoría de tos "idiopática" o "refractaria". 24

Una teoría alternativa, actualmente considerada, es que estos pacientes que permanecen con tos tienen una anormalidad en el reflejo tusígeno y que estas patologías podrían actuar como desencadenantes de la tos estimulando una hipersensibilidad refleja, similar a lo que ocurre con el reflejo del dolor ${ }^{25}$ (figura 2). Del mismo modo, algunos individuos a los que no se les encuentra la causa de la tos, pueden tener una híper reflexiva tusígena que les condiciona el síntoma por tiempo prolongado ${ }^{26}$.

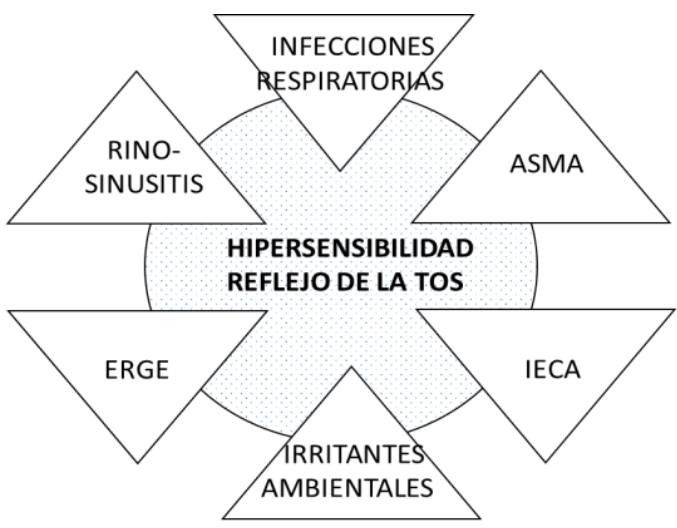

(TRPV1: potencial transitorio del receptor vanilloide tipo1; TRPA1: potencial transitorio del receptor ankirina tipo1; P2X2/3: subunidades 2 y 3 del receptor purinérgico; RAR: Receptor de adaptación rápida; RAL: Receptor de adaptación lenta).

Figura 1: Anatomía del reflejo de la tos
Así recientemente se reconoció la existencia de un síndrome de "tos por hipersensibilidad refleja o neuropatía" que se sustentó inicialmente en términos teóricos, pero que ha tenido considerables avances en su conocimiento fisiopatológico en los últimos años que le otorgan un soporte nosológico. ${ }^{26,27}$

\section{Tos por hipersensibilidad refleja.}

Se define como una entidad clínica caracterizada por tos desencadenada por bajos niveles de estímulos térmicos, mecánicos y químicos o situaciones tan habituales como hablar, cantar, el $\begin{array}{llll}\text { ejercicio } & y & \text { stress } & 25,27,28\end{array}$ El término se ha propuesto para identificar a un grupo de pacientes con tos crónica no explicada por una causa precisa, a los que no se les ha encontrado ninguna razón para justificar la prolongación de su síntoma utilizando el protocolo diagnóstico clásico y no se resuelve con un tratamiento etiológico ${ }^{28}$. Generalmente la tos es seca o escasamente productiva con disfonía y sensación urgente de toser. Los pacientes manifiestan una exagerada sensibilidad laríngea, donde se encuentran, en mayor número, los receptores de la tos ${ }^{27}$. Es más frecuente en mujeres, con un pico asociado al inicio de la menopausia, ${ }^{29,30}$ lo que podría estar asociado a factores epidemiológicos, biológicos y genéticos que condicionarían una mayor excitabilidad en el reflejo de la tos en mujeres de mayor edad $^{31}$. El principal mecanismo de la tos por hipersensibilidad refleja sería una alteración de la sensibilidad de receptores y vías sensoriales de la tos y del procesamiento central del reflejo de la $\operatorname{tos}^{32}$.

\section{Fisiopatología del reflejo de la tos}

Existen evidencias que sugieren que, en pacientes con tos de difícil control, a nivel central existiría una amplificación sostenida de los estímulos sensoriales aferentes y una reducida capacidad para suprimir los estímulos eferentes motores ${ }^{33}$. El mantenimiento de una repetida estimulación periférica puede conducir a un estado de hipersensibilidad central que puede ser reversible, aunque si la activación nociceptiva persiste se puede transformar en permanente ${ }^{32}$. La hipersensibilidad periférica puede ser la causa principal de la tos, pero la central puede

Revista Methodo: Investigación Aplicada a las Ciencias Biológicas. Universidad Católica de Córdoba Jacinto Ríos 571 Bo Gral. Paz. X5004FXS. Córdoba. Argentina. Tel.: (54) 3514517299 / Correo: methodo@ucc.edu.ar / Web: methodo.ucc.edu.ar | ARTICULO DE REVISION Methodo 2018;3(4):120- 
desencadenar un mecanismo de retroalimentación que mantiene la primera ${ }^{12}$

\section{Hiperactivación periférica}

El primer mecanismo responsable de tos es la excesiva sensibilidad de las terminaciones nerviosas periféricas a estímulos variados ${ }^{12}$.

Pareciera que diversos estímulos como irritantes, cambios de temperatura y virus producen cambios fenotípicos en las neuronas receptivas del reflejo tusígeno contribuyendo a una sobrestimulación. Estudios en cobayos demostraron que la aplicación intranasal del virus de la parainfluenza $3 \mathrm{y}$ rinovirus ${ }^{34}$ produjo un significativo aumento de la expresión del receptor TRPV1 de neuronas que contienen fibras C. Esto puede inducir la sobreproducción de neuropéptidos como sustancia $\mathrm{P}$ y péptido relacionado genéticamente a la calcitonina (CGRP) que favorece una inflamación neuroinmune con polarización hacia $\mathrm{Th} 2{ }^{35}$. En caso de persistencia de los desencadenantes, principalmente virus, se producirá un círculo vicioso permanente de hiperrespuesta tusígena ${ }^{32}$. Estudios clínicos en seres humanos demostraron que sujetos con infección respiratoria viral manifiestan un umbral de reactividad más bajo al desafío con capsaicina aunque no está demostrado en forma definitiva si es consecuencia del mecanismo desarrollado más arriba en modelos animales ${ }^{36,37}$. Se postula que la reducción en los niveles de estrógenos podría inducir una sobreexpresión del receptor TRPV1 y potenciar el reflejo tusígeno; ésta sería una de las razones del predominio del síndrome de tos por hipersensibilidad refleja en mujeres a mayor edad 31. La inflamación subclínica de la vía aérea también podría incrementarse en la menopausia.

\section{Alteraciones en los núcleos centrales integradores del reflejo}

Dos situaciones fisiopatológicas a nivel central parecen co-existir en la tos por hipersensibilidad refleja: por un lado, se manifiesta una activación de áreas del cerebro medio relacionadas a vías moduladoras descendentes y por otro, una activación disminuida de las áreas centrales relacionadas a la supresión de la tos ${ }^{33}$.

Con la inhalación de estímulos tusígenos, como capsaicina y ácido cítrico, bajo condiciones controladas en pacientes con tos crónica, el estudio por resonancia magnética funcional cerebral y tomografía con emisión de positrones reveló una sobre estimulación de los núcleos mediales cerebrales lo que explica una alteración del control del proceso neural central de la tos, evento más evidente en mujeres que en hombres ${ }^{38}$. Por otro lado, existe una pérdida del control inhibitorio en

áreas cerebrales ligadas a la supresión de la tos ${ }^{33,39}$ lo que también contribuye a la hiperexitabilidad refleja. La morfina ${ }^{40}$ tendría un efecto central agonista en estos últimos centros propiciando una recuperación de la función inhibitoria.

\section{Nuevos horizontes en el tratamiento de la tos refractaria}

Según los algoritmos emanados de opiniones de consenso, el objetivo prioritario para el abordaje de la tos crónica sigue siendo la identificación de la causa y su tratamiento específico 3,4,16,18,20,21. No obstante, dadas las características de la tos por hipersensibilidad refleja, en muchos casos, será necesario lograr la normalización de la hipersensibilidad central y periférica para contribuir a la supresión de la tos ${ }^{41}$.

Estudios preclínicos en animales ${ }^{42,43}$ arrojaron resultados promisorios, pero, curiosamente, los efectos han sido decepcionantes o contradictorios en los ensayos clínicos en humanos ${ }^{44}$. Por lo tanto, en la actualidad, las opciones para el tratamiento sintomático de la tos tanto en niños como adultos siguen siendo limitadas.

La morfina actúa en forma no selectiva sobre el sistema nervioso central y produce una significativa reducción de los puntajes sintomáticos de tos; no obstante, por sus características de opiáceo tiene considerables efectos adversos que no hacen propicia su utilización, especialmente en niños, y existe considerable variación en la respuesta entre individuos ${ }^{40}$ La codeína es uno de los más potentes supresores de la tos, pero por ser narcótico, al igual que la morfina, debe usarse el menor tiempo posible porque puede causar adicción, sedación, mareos, intolerancia digestiva y sequedad bucal ${ }^{40}$.

El dextrometorfano, un antitusivo no narcótico, equipotente con codeína y uno de los antitusivos más usados mundialmente como medicamento de venta libre, tiene efecto inhibidor del receptor $\mathrm{N}$ metil-D-aspartato (NMDA) a nivel central, pero, frecuentemente genera molestias gastrointestinales 45 .

El tratamiento con gabapentina, un inhibidor de los canales del calcio a nivel central con efecto regulador de la liberación de neurotransmisores, inicialmente utilizado como anticonvulsivante, 
logró mejorar la calidad de vida específica relacionada a la tos. Su dosificación debe ser individualizada y no está exenta de efectos adversos como mareos, inestabilidad corporal, depresión y comportamiento suicida ${ }^{46}$. Otra limitación de los opiáceos y gabapentina es que no suprimen la sensibilidad de receptores periféricos a capsaicina por lo que su efecto se ve atenuado si no se evitan o controlan los desencadenantes del reflejo tusígeno.

Otros inmunomoduladores como la pregabalina 47 y amitriptilina ${ }^{48}$ también han sido estudiados en pacientes adultos con tos refractaria con resultados disímiles y no exentos de efectos indeseables.

Están en desarrollo nuevas drogas con potencial efecto antitusivo, pero sus resultados clínicos al momento son contradictorios al considerar la ecuación riesgo (efectos adversos) y beneficio clínico. Así, se están investigando nuevas drogas con efecto blanco en los receptores periféricos y centrales del reflejo de la tos como P2X3, neurokinin-1, TRPV1, TRPV4 y TRPA1 41. Un reciente estudio doble ciego con control placebo en pacientes adultos con tos refractaria, un antagonista del receptor purinérgico $\mathrm{P} 2 \mathrm{X} 3$, el AF219/MK7264, logró una reducción del 75\% en la frecuencia de accesos de tos en relación al placebo, aunque su potencial utilidad clínica aun es incierta ${ }^{49}$.

El antagonista SB-705498 del receptor TRPV1 permitió una reducción de la sensibilidad refleja a la capsaicina, pero con escasa mejoría de los parámetros clínicos de la tos ${ }^{50}$. Otro antagonista de ese receptor, el XEN-D0501, tuvo mayor eficacia que el SB-705498 en inhibir la tos desencadenada por el desafío con capsaicina, pero falló en reducir la tos espontánea de los pacientes estudiados ${ }^{51}$. Esto indicaría que el abordaje de la tos refractaria no sólo debiera abarcar el bloqueo de receptores periféricos sino modular conjuntamente la hipersensibilidad de los núcleos centrales integradores del reflejo para lograr un control más efectivo del síntoma ${ }^{52}$.

\section{Conclusiones y perspectivas futuras}

La tos de evolución crónica sigue siendo un desafío médico con alto impacto clínico y social. El diagnóstico etiológico y la correspondiente resolución de la causa sigue siendo la regla de oro para un abordaje correcto. No obstante, muchos pacientes desarrollan un estado de hipersensibilidad refleja que condiciona la persistencia del síntoma a pesar de la resolución de la causa. Los recientes avances en el conocimiento de la fisiopatología de la tos ha llevado a considerar a la tos por hipersensibilidad refleja como una entidad clínica, muchas veces paralela, que requiere un abordaje distinto al paradigma etiológico antes predicado por los consensos y guías clínicas. A pesar de las nuevas investigaciones farmacológicas se necesitan evidencias más seguras para aconsejar un tratamiento "sintomático" de estos pacientes que contribuya al control de la hipersensibilidad central y periférica del reflejo de la tos.

\section{Bibliografía}

1. French CL, Irwin RS, Curley FJ, Krikorian CJ. Impact of chronic cough on quality of life. Arch Intern Med 1998; 158:1657-61.

2. Young EC, Smith JA. Quality of life in patients with chronic cough. Ther Adv Respir Dis 2010; 4:49-55.

3. Irwin RS, Baumann MH, Bolser DC, Boulet LP, Braman SS, Brightling CE, et al. Diagnosis and management of cough executive summary: ACCP evidence-based clinical practice guidelines. Chest 2006;129 (suppl):1S-23S

4. Shields MD, Bush A, Everard M L, McKenzie S, Primhak R, et al. Recommendations for the assessment and management of cough in children. Thorax 2008;63(Suppl. III): iii1iii15.

5. O'Grady KF, Drescher BJ, Goyal V, Phillips $\mathrm{N}$, Acworth J, Marchant JM, Chang AB. Chronic cough postacute respiratory illness in children: a cohort study. Arch Dis Child 2017; 102:1044-48

6. Marchant JM, Newcombe PA, Juniper EF, Sheffield JK, Stathis SL, Chang AB. What is the burden of chronic cough for families? Chest 2008; 134:303-9.

7. McGarvey LP. Are there clinical features of a sensitized cough reflex? Pulm Pharmacol Ther 2009;22: 59-64.

8. Chung KF, Pavord ID. Prevalence, pathogenesis, and causes of chronic cough. Lancet 2008;371: 1364-74.

9. Chang $\mathrm{AB}$, Berkowitz RG. Cough in the pediatric population. Otolaryngol Clin N Am 2010; 43:181-98

10. Canning BJ. Anatomy and neurophysiology of the cough reflex: ACCP evidence-based clinical practice guidelines. Chest 2006; 129:33-47.

11. Canning BJ, Chang AB, Bolser DC, Smith JA, Mazzone SB, McGarvey L. Anatomy

Revista Methodo: Investigación Aplicada a las Ciencias Biológicas. Universidad Católica de Córdoba. Jacinto Ríos 571 Bo Gral. Paz. X5004FXS. Córdoba. Argentina. Tel.: (54) 3514517299 / Correo: methodo@ucc.edu.ar / Web: methodo.ucc.edu.ar | ARTICULO DE REVISION Methodo 2018;3(4):120- 
and neurophysiology of cough:

Chest guideline and expert panel report.

Chest 2014; 146:1633-48.

12. Canning BJ. Afferent nerves regulating the cough reflex: mechanisms and mediators of cough in disease. Otolaryngol Clin North Am 2010; 43:15-25.

13. West PW, Canning BJ, Merlo-Pich E, Woodcock AA, Smith JA. Morphologic characterization of nerves in whole-mount airway biopsies. Am J Respir Crit Care Med 2015; 192:30- 9 .

14. Chen CY, Bonham AC, Schelegle ES, Gershwin LJ, Plopper CG, Joad JP. Extended allergen exposure in asthmatic monkeys induces neuroplasticity in nucleus tractus solitarius. J Allergy Clin Immunol 2001; 108:557-62.

15. McGovern AE, Driessen AK, Simmons DG. Distinct brainstem and forebrain circuits receiving tracheal sensory neuron inputs revealed using a novel conditional anterograde transsynaptic viral tracing system. J Neurosci 2015;35: 7041-55.

16. Irwin RS, Curley FJ, French CL. Chronic cough: The spectrum and frequency of causes, key components of the diagnostic evaluation and outcome of specific therapy. Am Rev Respir Dis 1990; 141:640-7.

17. Chang AB, Glomb WB.Guidelines for evaluating chronic cough in pediatrics: ACCP Evidence-Based Clinical Practice Guidelines. Chest 2006; 129:260S-283S.

18. Morice AH, McGarvey L, Pavord I. Recommendations for the management of cough in adults. Thorax 2006;61(suppl 1): i124.

19. Morice AH, Fontana GA, Belvisi MG, et al. ERS guidelines on the assessment of cough. Eur Respir J 2007; 29:1256-76.

20. Gibson P, Chang A, Glasgow N, et al. Cough in children and adults, diagnosis and assessment: Australian cough guidelines. Med J Aust 2010; 192:265-71.

21. Saranz RJ, Lozano A, Lozano N, Castro Rodríguez JA. Diagnóstico y tratamiento de la tos crónica en pediatría. Arch Argent Pediatr 2013; 111:140-7.

22. Faniran AO, Peat JK, Woolcock AJ. Persistent cough: is it asthma? Arch Dis Child 1998; 79:411-4.
23. O'Hara J, Jones NS. "Post-nasal drip syndrome": most patients with purulent nasal secretions do not complain of chronic cough. Rhinology 2006; 44:270-3.

24. Haque RA, Usmani OS, Barnes PJ. Chronic idiopathic cough: ¿a discrete clinical entity? Chest 2005; 127:1710-3.

25. Song WJ, Chang YS, Morice AH. Changing the paradigm for cough: does 'cough hypersensitivity' aid our understanding?. Asia Pac Allergy 2014; 4:3-13.

26. Chung KF. Chronic 'cough hypersensitivity syndrome': a more precise label for chronic cough. Pulm Pharmacol Ther 2011; 24:26771.

27. Song WJ, Morice AH. Cough hypersensitivity syndrome: A few more steps forward. Allergy Asthma Immunol Res 2017; 9:394-402.

28. Morice AH, Millqvist E, Belvisi MG, Bieksiene K, Birring SS, Chung KF, et al. Expert opinion on the cough hypersensitivity syndrome in respiratory medicine. Eur Respir J 2014; 44:1132-48.

29. Everett C, Kastelik J, Thompson R, et al. Chronic persistent cough in the community: a questionnaire survey. Cough 2007;3:5.

30. Morice AH, Jakes AD, Faruqi S, Birring SS, McGarvey L, Canning B, Smith JA. A worldwide survey of chronic cough: a manifestation of enhanced somatosensory response. Eur Respir J 2014; 44:1149-55.

31. Plevkova J, Buday $\mathrm{T}$, KavalcikovaBogdanova N, Ioan I, Demoulin-Alexikova S. Sex differences in cough reflex. Respir Physiol Neurobiol 2017; 245:122-9.

32. Pacheco A. Chronic cough: from a complex dysfunction of the neurological circuit to the production of persistent cough. Thorax 2014;69: 881-3.

33. Ando A, Smallwood D, McMahon M, Irving L, Mazzone SB, Farrell MJ Neural correlates of cough hypersensitivity in humans: evidence for central sensitisation and dysfunctional inhibitory control. Thorax 2016;71: 323-9.

34. Abdullah H, Heaney LG, Cosby SL, McGarvey LP. Rhinovirus upregulates transient receptor potential channels in a human neuronal cell line: implications for respiratory virus-induced cough reflex sensitivity. Thorax 2014;69: 46-54.

35. Song WJ, Chang YS. Cough hypersensitivity as a neuro-immune interaction. Clin

Revista Methodo: Investigación Aplicada a las Ciencias Biológicas. Universidad Católica de Córdoba. Jacinto Ríos 571 Bo Gral. Paz. X5004FXS. Córdoba. Argentina. Tel.: (54) 3514517299 / Correo: methodo@ucc.edu.ar / Web: methodo.ucc.edu.ar | ARTICULO DE REVISION Methodo 2018;3(4):120- 
Translational Allergy 2015.DOI 10.1186/s13601-015-0069-4

36. O'Connell F, Thomas VE, Studham JM, Pride NB, Fuller RW. Capsaicin cough sensitivity increases during upper respiratory infection. Respir Med 1996;90: 279- 86.

37. Dicpinigaitis PV, Bhat R, Rhoton WA, Tibb AS, Negassa A. Effect of viral upper respiratory tract infection on the urge-tocough sensation. Respir Med 2011; 105: 6158 .

38. Mazzone SB, Cole LJ, Ando A, Egan GF, Farrell MJ. Investigation of the neural control of cough and cough suppression in humans using functional brain imaging. $\mathrm{J}$ Neurosci 2011;31: 2948-58.

39. Mazzone S, McGovern AE, Yang S-K, et al. Sensorimotor circuitry involved in the higher brain control of coughing. Cough 2013; 9:7.

40. Morice AH, Menon MS, Mulrennan SA, et al. Opiate therapy in chronic cough. Am J Respir Crit Care Med 2007;175: 312-15.

41. Keller JA, McGovern AE,Mazzone SB. Translating cough mechanisms into better cough suppressants. Chest 2017; 152:833-41.

42. Belvisi MG, Patel HJ, Freund-Michel V, Hele DJ, Crispino N, Birrell MA. Inhibitory activity of the novel CB2 receptor agonist, GW833972A, on guinea pig and human sensory nerve function in the airways. $\mathrm{Br} \mathbf{J}$ Pharmacol 2008;155: 547- 57.

43. Delescluse I, Mace H, Adcock JJ. Inhibition of airway hyper-responsiveness by TRPV1 antagonists (SB-705498 and PF-04065463) in the unanaesthetized, ovalbumin-sensitized guinea pig. Br J Pharmacol 2012; 166:182232.

44. Jolley CJ, Birring SS. New drug targets for chronic cough: research you can literally sink your teeth into! Eur Respir J 2017; 50: 1701571 .

45. Schaefer MK, Shehab N, Cohen AL, Budnitz DS. Adverse events from cough and cold medications in children. Pediatrics 2008;121: 783-7.

46. Ryan NM, Birring SS, Gibson PG. Gabapentin for refractory chronic cough: a randomised, double-blind, placebo-controlled trial. Lancet 2012; 380:1583-89.

47. Vertigan AE, Kapela SL, Ryan NM, et al. Pregabalin and speech pathology combination therapy for refractory chronic cough: a randomized controlled trial. Chest 2016; 149: 639-48

48. Jeyakumar A, Brickman TM, Haben M. Effectiveness of amitriptyline versus cough suppressants in the treatment of chronic cough resulting from postviral vagal neuropathy. Laryngoscope 2006; 116: 2108-112.

49. Abdulqawi R, Dockry R, Holt K, et al. P2X3 receptor antagonist (AF-219) in refractory chronic cough: a randomised, double-blind, placebo-controlled phase 2 study. Lancet 2015;385: 1198-1205.

50. Khalid S, Murdoch R, Newlands A, et al. Transient receptor potential vanilloid 1 (TRPV1) antagonism in patients with refractory chronic cough: a double-blind randomized controlled trial. J Allergy Clin Immunol 2014;134: 56-62.

51. Belvisi MG, Birrell MA, Wortley MA, Maher SA, Satia I, Badri H, Holt K, Round P, McGarvey L, Ford J, Smith JA. XEN-D0501, a novel TRPV1 antagonist, does not reduce cough in refractory cough patients. Am J Respir Crit Care Med 2017; 196:1255-63.

52. Ryan NM, Vertigan AE, Birring SS. An update and systematic review on drug therapies for the treatment of refractory chronic cough. Expert Opin Pharmacother 2018; 19:687-711.

\section{Palabras claves:}

TOS, HIPERSENSIBILIDAD, FISIOPATOLOGÍA, ANTITUSIVOS.

\section{Keywords:}

COUGH, HYPERSENSITIVITY, PATHOPHYSIOLOGY, COUGH SUPPRESSANTS.

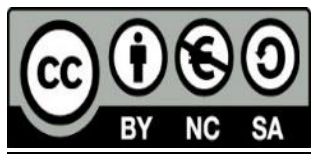

Revista Methodo: Investigación Aplicada a las Ciencias Biológicas. Universidad Católica de Córdoba. Jacinto Ríos 571 Bo Gral. Paz. X5004FXS. Córdoba. Argentina. Tel.: (54) 3514517299 / Correo: methodo@ucc.edu.ar / Web: methodo.ucc.edu.ar | ARTICULO DE REVISION Methodo 2018;3(4):120- 